\title{
PATTERNING OF CONDUCTIVE NANO-LAYERS ON GARNET
}

\author{
1'Jana CHLUMSKÁ, Ondřej LALINSKÝ, Milan MATĚJKA, Stanislav KRÁTKÝ, Vladimír KOLAŘíK \\ ${ }^{1}$ Institute of Scientific Instruments of CAS, Brno, Czech Republic, EU, chlumska@isibrno.cz
}

https://doi.org/10.37904/nanocon.2020.3731

\begin{abstract}
Synthetic crystalline materials of the garnet group are used as scintillators in scanning electron microscopy. If a thick conductive layer is applied on the garnet surface, slower electrons don't have enough energy to pass through this relatively thick conductive layer on the scintillator surface. Therefore, either thinner conductive layer or appropriate patterning of the thicker layer has to be used. Within this contribution we study the patterning process of such conductive nano-layer. Resolution of the patterning process is of high interest. Two approaches are compared: direct writing electron beam lithography and mask projection UV lithography.
\end{abstract}

Keywords: Electron beam lithography, nano-patterning, yttrium aluminium garnet

\section{INTRODUCTION}

Scintillation detectors are one of the most widely used types of backscattered electron (BSE) and secondary electron (SE) detectors. Their basic element is a scintillator. The scintillator is a material in which a cathodoluminescence $(\mathrm{CL})$ response occurs after an electron impact. The integral intensity of $\mathrm{CL}$ is a very important physical quantity and its magnitude affects the performance of the overall scintillation detector. The problem arises in the detection of low-energy electrons, since the integral intensity of $\mathrm{CL}$ decreases significantly with decreasing energy of incident electrons. SEs, having an energy of the order of tens of eV, are accelerated by the applied positive voltage on the scintillator, so that upon impact on the scintillator they elicit an intense CL response. BSEs have an energy close to that of the primary beam and are often detected by a scintillator without a positive potential applied. When the low energy of the primary beam is selected, the detected BSEs also have a low energy, which causes a weak CL response.

Another important factor influencing the $\mathrm{CL}$ response is the depth of electron penetration into the scintillator, which also decreases significantly with decreasing energy of the incident electrons. Then in single-crystalline scintillators, CL does not occur deep in the bulk, but closer to the surface of the scintillator. The energy quantum states at the surface do not have to create radiative recombination centers as in the bulk, but can change their nature from radiative to non-radiative. In addition to the surface treatment of the scintillator after its growth (cutting, grinding, polishing, thermochemical treatment), it also depends on the chemical composition and crystal structure of the scintillator. For the detection of electrons with an energy in hundreds of eV, a completely different scintillator may be suitable due to its $\mathrm{CL}$ response than for the detection of electrons with an energy of $10 \mathrm{keV}$.

Various approaches were presented for the etching of the ITO (Tin-doped indium oxide) conductive layer [1-3]. Alternatively, highly luminescent thin films of zinc tungstate have been deposited on top of conventional scintillators for electron detection in order to replace the need for a top conducting layer [4].

The aim of this contribution is to develop a technology for patterning of an ITO nano-layer on a single crystalline scintillator substrate. Microlithography shall be used for this purpose, both direct write e-beam lithography and mask projection UV lithography (using the EBL originated photo mask) are of interest. Pattern line width is expected in the range 1-2 microns; the final surface coverage of the patterned ITO layer should be less than $20 \%$. The ITO layer with specific parameters is coated on the sample surface before the lithography steps. 


\section{METHOLOGICAL BASIS}

Basically, a lithographic process is used for patterning of the thin conductive layer. The electron-beam directwrite patterning generally provides higher resolution and flexibility. The advantage of the optical projection lithography is basically lower processing cost.

\section{EXPERIMENTS}

The garnet sample (Figure 1, left) was coated by a conductive ITO layer of 30-50 nm. A specific sample holder was designed for the spin coating process (Figure 1, right).
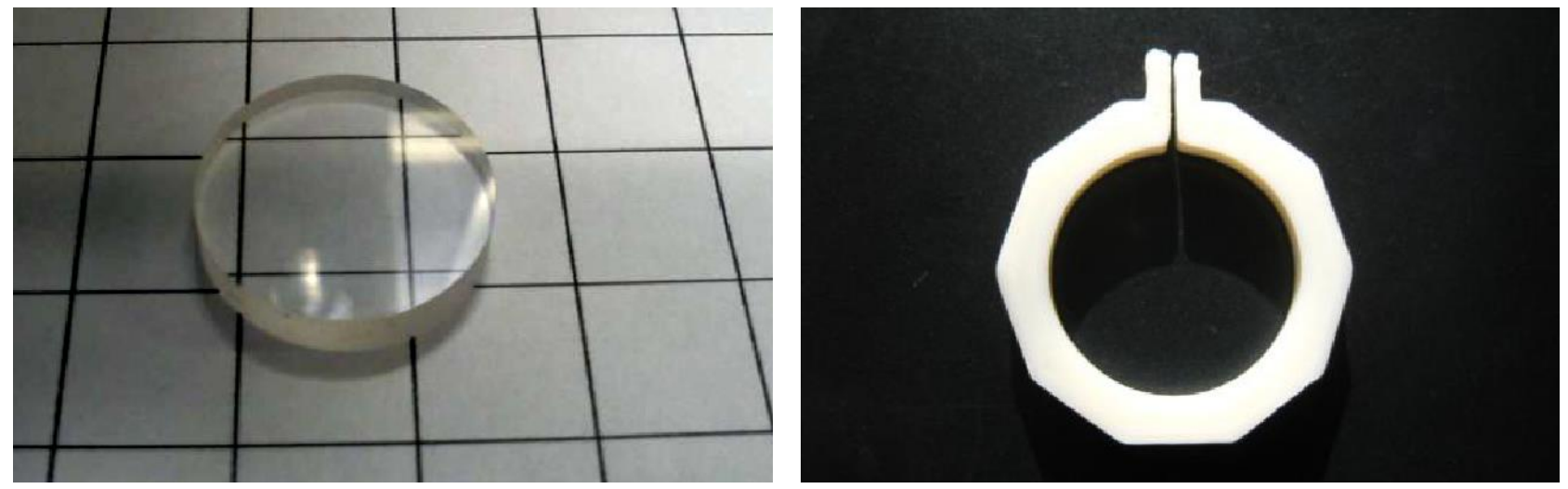

Figure 1 Garnet sample (left); sample holder for spin-coating process

\subsection{Direct Writing Electron Beam Lithography}

First, the sample was coated by an electron resist layer. Two types of positive-tone resist were evaluated, PMMA and Shipley S1813. The adhesivity of both types on the ITO layer was very poor. Known approaches for improving the adhesivity, i.e. plasma treating and adhesive promoter, were not effective. The resist layer was peeled off, partially or fully, either during the resist development process or during the ITO etching process. Partial results were achieved only for rough patterns; testing pattern in successive technological steps is shown in Figure 2. Moreover, the positive tone resist is not very suitable for the intended grid pattern, due to proximity effect of back scattered electrons during the exposure, the technological window of the applied dose is very narrow. The appropriate etching of the ITO layer was achieved using the $\mathrm{HCl}+\mathrm{H}_{2} \mathrm{O} 1: 1$ solution.
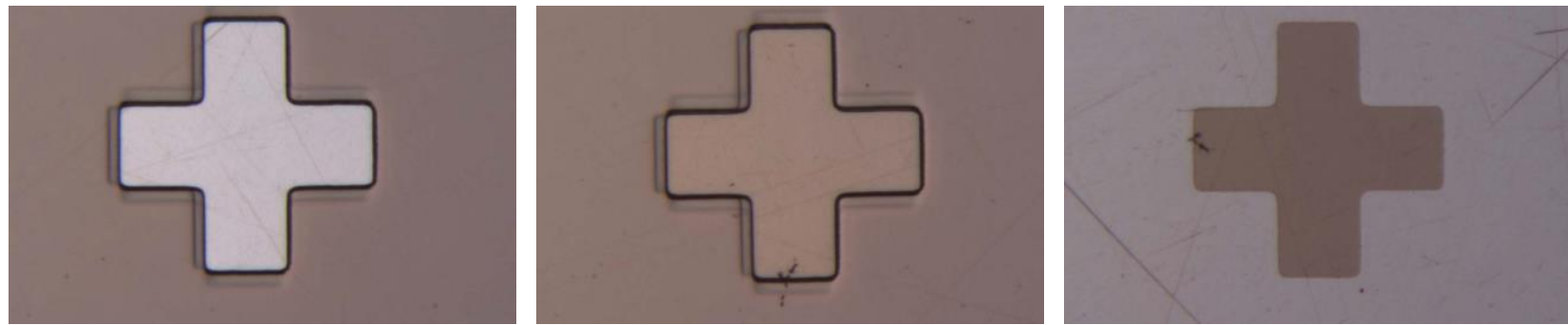

Figure 2 Sample test mark (see text): after resist development (left), after wet etching (center), after resist removing (right)

\subsection{Mask Projection UV Lithography}

The resolution of grid patterns was aimed at line width of less than 2 microns, with the spacing between lines in order of 20 microns; the final coverage of the ITO layer should be in the range $10-20 \%$. 
The projection photo mask was prepared by using the e-beam lithography. The glass substrate was covered by the chromium layer of $100 \mathrm{~nm}$ and subsequently by a PMMA resist layer of $400 \mathrm{~nm}$. The patterning was performed by using the e-beam pattern generator Vistec EBPG5000+ES with $100 \mathrm{keV}$ energy of electrons. After the development of the resist mask, the pattern was transferred into the chromium layer by wet etching process, using a commercial solution TechniStrip from MicroChemicals. Detail of the photo mask is depicted in Figure 3.

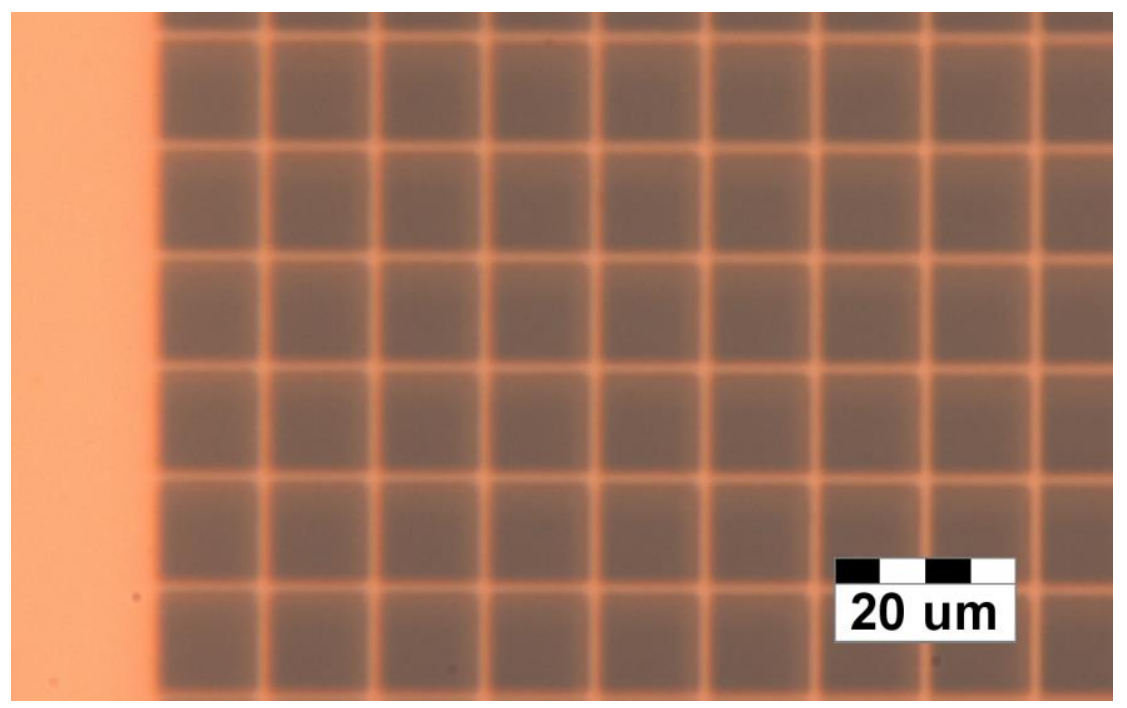

Figure 3 Chromium photo mask (light microcopy); grid size 12 um; line width 2.0 um

The garnet sample with the ITO layer was coated by $200 \mathrm{~nm}$ layer of the photoresist AZ 1518 from MicroChemicals. The photo lithography transfer of pattern from the mask to the photoresist was done by using the mask aligner MA/BA6 form Süss. The exposure time was set to 7 seconds; the resist was developed by AZ 726 MIF developer for 15 seconds. The ITO layer was etched with $\mathrm{HCl}+\mathrm{H}_{2} \mathrm{O}$ solution $1: 1$ for 30 seconds. The photo resist adhesion was found to be acceptable.

\section{RESULTS AND DISCUSSION}

The designed mask line of 3 microns was transferred as 1.5-2.0 micron lines in the ITO layer, thus achieving the final line width below 2 micrometers. The AFM measurements are shown in Figure 4; the micro photo is shown in Figure 5. The surface electrical conductivity of the sample was successfully checked.

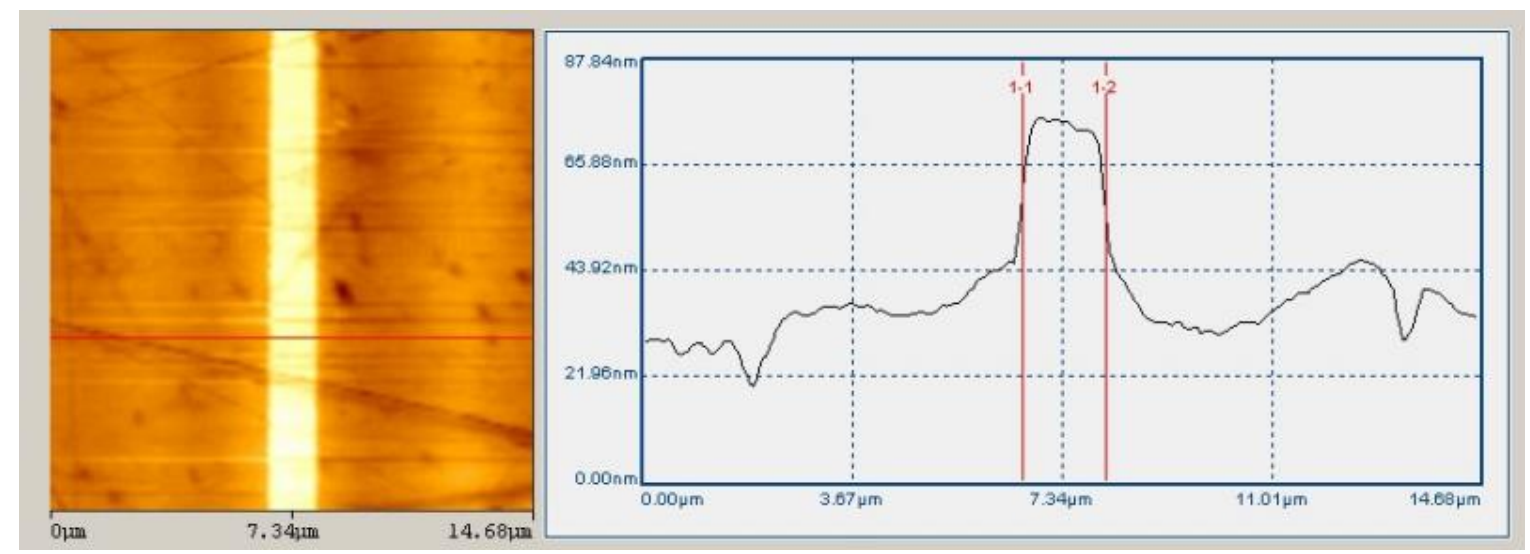

Figure 4 Conductive line on garnet (AFM); grid size 20 um; line width 1.5 um; thickness $~ 30 \mathrm{~nm}$ 


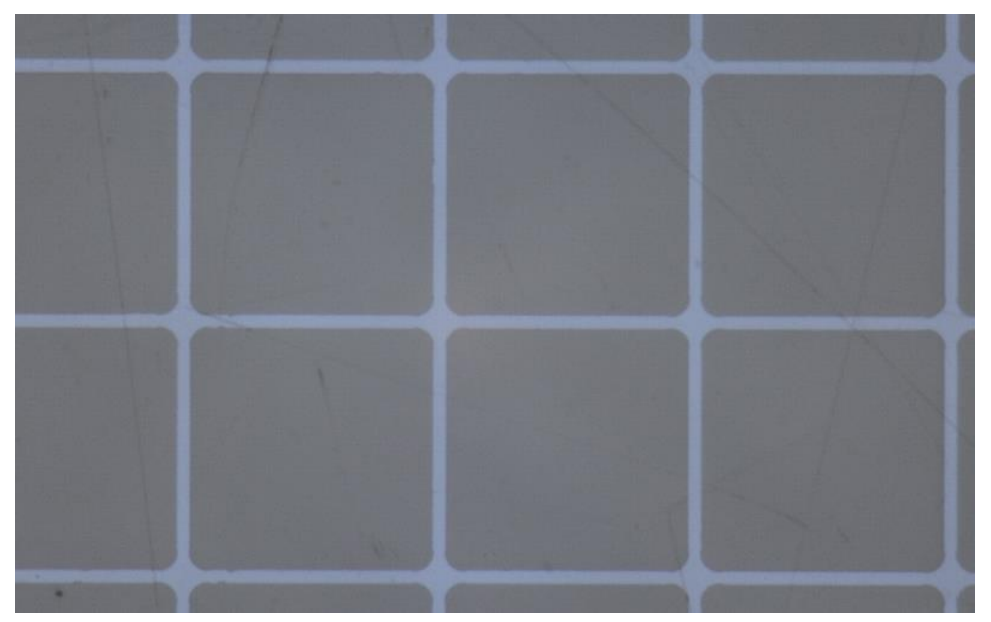

Figure 5 Conductive grid-patterned nano layer on garnet (light microcopy); grid size 20 um; line width 1.5 um; thickness $~ 30 \mathrm{~nm}$

\section{CONCLUSIONS}

The paper summarizes the results of patterning conductive nano layer on the garnet substrate. Direct write lithography using electron beam patterning was unsuccessful. The projection optical lithography using the ebeam originated photo mask was shown to be adequate and precise process. The resulting sample is used as a cathodoluminiscence detector with defined surface conductivity.

\section{ACKNOWLEDGEMENTS}

The research was funded by the Technology Agency of the Czech Republic (project TN01000008). The research infrastructure was funded by the Czech Academy of Sciences (project RVO:68081731).

\section{REFERENCES}

[1] TSAI, T.-H., WU, Y.-F. Wet etching mechanisms of ITO films in oxalic acid. Microelectronic Engineering. March 2006, vol. 83, no. 3, pp 536-541.

[2] HUANG, C.J., SU, Y.K., WU, S.L. The effect of solvent on the etching of ITO electrode. Materials Chemistry and Physics. March 2004, vol. 84, no. 1, pp. 146-150.

[3] SCHOLTEN, M., MEERAKKER, J.E.A.M. van den. On the Mechanism of ITO Etching: The Specificity of Halogen Acids. J. of the Electrochemical Society. 1993, vol. 140, no. 2, pp. 471-475.

[4] TZOLOV, M. B. et al. First-Surface Scintillator for Low Accelerating Voltage Scanning Electron Microscopy (SEM). Imaging. Microscopy and Microanalysis. 2018, vol. 24, no. 5, pp. 488-496. 